\title{
Partial Derivatives of Eigenfrequencies of a Rectangular Parallelepiped and a Sphere of Elastically Anisotropic Solid
}

\author{
Hitoshi Oda, ${ }^{1, *}$ Isao Suzuki, ${ }^{1}$ and Ichiro Ohno ${ }^{2}$ \\ ${ }^{1}$ Faculty of Science, Okayama University, Okayama 700, Japan \\ ${ }^{2}$ Faculty of Science, Ehime University, Matsuyama 790, Japan
}

\begin{abstract}
Analytic expressions of partial derivatives of eigenfrequency with respect to elastic constants, density and size were derived for a rectangular parallelepiped and a sphere of elastically anisotropic solid, in order to provide an accurate inversion method for determining the elastic constants. The formulas were extended for determination of anisotropic dissipation constants from attenuation data of free oscillation of anelastic materials. A numerical example was given for an olivine specimen (orthorhombic) of rectangular parallelepiped.
\end{abstract}

\section{Introduction}

Elasticity measurement of Earth's materials is important for studies on constitution and structure of the Earth. Many methods have been devised for determination of elastic constants of the materials, most of which are macroscopically and microscopically anisotropic. The interferometry is excellent in accuracy (McSkimin, 1964), although a rather large specimen or plural ones are needed for measurements at different orientations, in particular, for low symmetry crystals. The Brillouin scattering method is also useful for very small specimens (Weidner and Carleton, 1977; Sawamoto et al., 1984), but small defects in the materials often make the elasticity measurement difficult.

In the past decade, the resonance method was developed for accurate determination of elastic constants of a small specimen and it was applied to a sphere and a cube of isotropic solid (Soga and Anderson, 1967; Demarest, 1971), and also to a rectangular parallelepiped of anisotropic solids with orthorhombic crystal symmetry (Ohno, 1976) and with trigonal crystal symmetry (Ohno et al., 1986). In the resonance method; we measure only the lowermost frequencies on one piece of small specimen, so that the applicability has been widely extended to the materials of geophysical interests. In fact, the rectangular parallelepiped resonance (RPR) method has been used to determine elastic constants of a number of rock-forming minerals of geophysical interest (Goto et al., 1976; Sumino et al., 1976; Sumino and Nishizawa, 1978; Babuska et al., 1978;

Received April 6, 1993; Accepted September 30, 1993

* To whom correspondence should be addressed. 
Suzuki et al., 1983; Yamamoto et al., 1987). The resonance method has merits in applicability to specimen with various shapes and sizes in principle, and also in measurements at low temperatures to $80 \mathrm{~K}$ (Sumino et al., 1983) and at high temperatures even up to $1,800 \mathrm{~K}$ (Goto and Anderson, 1988). The resonant sphere technique (RST) used for elasticity measurement of isotropic material (Soga and Anderson, 1967) may also have potential in geophysics, if its applicability could be extended to anisotropic materials.

The resonance method is an indirect one to determine elastic constants of a specimen through an inversion procedure, and the basic data acquired by measurement are the resonant frequencies of many oscillatory modes. An angular eigenfrequency, $\omega$, is dependent on all elastic constants $\left\{C_{i}\right\}$, mass $M$ and shape of the specimen

$$
\omega=\omega\left(C_{i}, M, s_{j}\right),
$$

where $s_{j}$ denotes the parameters specifying the shape and size of the specimen. In the resonance method, we measure resonant frequencies of many eigenmodes and sizes $\left\{s_{j}\right\}$ for a specimen with a mass $M$, and determine $\left\{C_{i}\right\}$ in order that the observed eigenfrequencies satisfy Eq. (1). This is a typical inversion problem to be solved with the least squares optimization. In the inversion calculation, the observation equations are expressed as

$$
\delta \omega=\omega^{\circ}-\omega^{c}=\left(\partial \omega^{\mathrm{c}} / \partial C_{i}^{\left({ }^{(r)}\right.}\right)\left\{C_{i}-C_{i}^{(r)}\right\}+\left(\partial \omega^{c} / \partial s_{j}^{(r)}\right)\left\{s_{j}-s_{j}^{(r)}\right\}
$$

where $\omega^{\circ}$ is the observed angular eigenfrequency, $\omega^{\mathrm{c}}$ is the computed one by using the elastic constants $\left\{C_{i}^{(\mathrm{r})}\right\}$ and size $\left\{s_{j}^{(\mathrm{r})}\right\}$ at a reference state (r), and $\left\{C_{i}\right\}$ are elastic constants to be determined. The last term in Eq. (2) is a symbolic expression of correction for eigenfrequency due to the small changes of shape and size of the specimen. In Eq. (2), the summation convention is assumed for the repeated indices, hereafter unless stated. In order to determine the $\left\{C_{i}\right\}$ from a set of Eq. (2) for many oscillatory modes, we have to evaluate accurately the partial derivatives, $\left(\partial \omega^{\mathrm{c}} / \partial C_{i}^{(\mathrm{r})}\right)$ and $\left(\partial \omega^{\mathrm{c}} / \partial s_{j}^{\left({ }^{(r)}\right.}\right)$, as well as the eigenfrequencies.

In the RPR method, the eigenfrequencies have been computed by the Rayleigh-Ritz method (variational method), in which the displacement eigenfunctions are expanded by the Legendre functions (cf., Demarest, 1971; Ohno, 1976). For computation of eigenfrequencies of an elastically anisotropic sphere by the Rayleigh-Ritz method, on the other hand, free oscillation eigenfunctions of a homogeneous and isotropic sphere are used as basis functions (Mochizuki, 1986, 1988). Thus, the numerical differentiation method is an easy way for evaluating the derivatives of eigenfrequency with respect to elastic constants. In the numerical method, a small change $\Delta \omega$ of eigenfrequency is computed for a small change $\Delta C_{i}$ of each elastic constant, and the derivative of eigenfrequency is numerically calculated by $\Delta \omega / \Delta C_{i}$. However, such derivatives may contain some errors due to nonlinearity between $\omega$ and $C_{i}$ and computational errors (deficiency of significant figures). Particularly, computation of the derivatives by the numerical differentiation method is not practical for free vibration of anisotropic sphere, because it takes a very long time to compute the eigenfrequencies. Thus, formulas to compute directly the derivatives are desired to be expressed and programmed, so that the partial derivatives are computed as soon as the eigenfrequencies are obtained. Such 
formulation has been known in seismology for isotropic or transversely isotropic Earth (Takeuchi and Saito, 1972), but it has not yet been obtained for general anisotropic materials with shape of sphere and rectangular parallelepiped.

The purpose of the present paper is to present theoretical background for computation of the derivatives, $\partial \omega / \partial C_{i}$ and $\partial \omega / \partial s_{j}$, for elastically anisotropic solids with general shape, and to obtain analytic expressions of the derivatives for a rectangular parallelepiped and a sphere. These formulas are also applied to description of anisotropic energy dissipation of material. A numerical example is given for an olivine of rectangular parallelepiped in shape.

\section{Partial Derivatives of Free Oscillation Eigenfrequency}

The free oscillation of an elastic body with volume $V$ is described by a variational equation in the Cartesian coordinates

$$
\delta \int L \mathrm{~d} V=0
$$

where the $L$ is Lagrangian defined by

$$
L=\varrho \omega^{2} U_{i}^{*} U_{i}-\varepsilon_{i j}^{*} C_{i j k l} \varepsilon_{k l} .
$$

The asterisk above denotes the complex conjugate, and $C_{i j k l}$ is the fourth-order elastic tensor. The $\varepsilon_{i j}$ is the strain tensor defined by $\left(U_{i, j}+U_{j, i}\right) / 2$, where $U_{i, j}$ is the spatial derivative of the $i$-th component $U_{i}$ of displacement vector $U$ with respect to $x_{j}$. The displacement vector $U$ is expressed by a linear combination of known basis function vectors $v^{(p)}$ as follows:

$$
U=\sum_{p=1}^{N} a_{p} v^{(p)}
$$

where $N$ is the number of basis functions, $a_{p}$ 's are the unknown constants to be determined, and the summation with respect to the index $p$ or $q$ is always explicitly shown through this paper to avoid confusion with the conventional expression. In order to determine the eigenfrequency $\omega$ as well as the unknown constants $a_{p}$, we substitute Eq. (5) into Eq. (3) with the aid of Eq. (4), and then obtain

$$
\sum_{q=1}^{N}\left(\omega^{2}\langle p|\varrho| q\rangle-\langle p|C| q\rangle\right) a_{q}=0, \quad(p=1,2,3, \cdots, N)
$$

where

$$
\begin{aligned}
& \langle p|\varrho| q\rangle=\int v_{i}^{(p) *} \varrho v_{i}^{(q)} \mathrm{d} V \\
& \langle p|C| q\rangle=\int \varepsilon_{i j}^{(p) *} C_{i j k l} \varepsilon_{k l}^{(q)} \mathrm{d} V
\end{aligned}
$$

Vol. 41, No. 5, 1993 
and $\varepsilon_{i j}^{(p)}$ is strain tensor for $v^{(p)}$. When we adopt a condition of constraint for the coefficients $a_{p}$ 's

$$
\sum_{p=1}^{N} a_{p}^{*} a_{p}=1
$$

Eq. (6) is an eigenvalue equation, where $\omega^{2}$ is an eigenvalue and the $a_{p}$ 's are the components of eigenvector corresponding to the eigenvalue. Once the eigenvalue and eigenvector are determined from Eq. (6), the angular eigenfrequency $\omega$ is obtained from the eigenvalue and the displacement vector $U$ is computed by substituting the eigenvector into Eq. (5).

From Eqs. (3) and (5), the energy equation of free oscillation is expressed as

$$
\sum_{p=1}^{N} \sum_{q=1}^{N}\left(\omega^{2}\langle p|\varrho| q\rangle-\langle p|C| q\rangle\right) a_{p}{ }^{*} a_{q}=0
$$

Now let us consider change $\delta \omega$ of $\omega$ due to small changes of density and elastic constants, $\delta \varrho$ and $\delta C_{i j k l}$. Because the displacement vector $U$ may also change into $U+\delta U$ owing to the $\delta \rho$ and $\delta C_{i j k l}$

$$
U+\delta U=\sum_{p=1}^{N} \cdot\left(a_{p}+\delta a_{p}\right) v^{(p)}
$$

the energy Eq. (9) is written as

$$
\sum_{p=1}^{N} \sum_{q=1}^{N}\left((\omega+\delta \omega)^{2}\langle p|\varrho+\delta \varrho| q\rangle-\langle p|C+\delta C| q\rangle\right)\left(a_{p}+\delta a_{p}\right)^{*}\left(a_{q}+\delta a_{q}\right)=0 .
$$

When the higher-order terms of $\delta \varrho, \delta \omega, \delta C_{i j k l}$, and $\delta a_{p}$ are ignored, the variational equation, i.e. the difference between Eqs. (9) and (11), is approximated as

$$
\begin{aligned}
& \sum_{p=1}^{N} \sum_{q=1}^{N}\left(\omega^{2}\langle p|\varrho| q\rangle-\langle p|C| q\rangle\right)\left(a_{p}{ }^{*} \delta a_{q}+a_{q} \delta a_{p}{ }^{*}\right) \\
& +\sum_{p=1}^{N} \sum_{q=1}^{N}\left(\omega^{2}\langle p|\delta \varrho| q\rangle+2 \omega \delta \omega\langle p|\varrho| q\rangle-\langle p|\delta C| q\rangle\right) a_{p}{ }^{*} a_{q}=0 .
\end{aligned}
$$

The first summation vanishes on account of Eq. (6), and the variational equation is reduced to

$$
\sum_{p=1}^{N} \sum_{q=1}^{N}\left(\omega^{2}\langle p|\delta \varrho| q\rangle+2 \omega \delta \omega\langle p|\varrho| q\rangle-\langle p|\delta C| q\rangle\right) a_{p}{ }^{*} a_{q}=0
$$

where the first and the last terms, respectively, represent the changes in kinetic and strain energies due to the small changes, $\delta \varrho$ and $\delta C_{i j k l}$. For a homogeneous and anisotropic body, the elastic constants and density are independent of coordinates, and they are moved outside the brackets, 


$$
\begin{gathered}
2 \omega \delta \omega \varrho \sum_{p=1}^{N} \sum_{q=1}^{N}\langle p \mid q\rangle a_{p}{ }^{*} a_{q}=-\omega^{2} \delta \varrho \sum_{p=1}^{N} \sum_{q=1}^{N}\langle p \mid q\rangle a_{p}{ }^{*} a_{q} \\
+\delta C_{i j k l} \sum_{p=1}^{N} \sum_{q=1}^{N}\langle p \mid q\rangle_{i j k l} a_{p}{ }^{*} a_{q},
\end{gathered}
$$

where

$$
\begin{aligned}
& \langle p \mid q\rangle=\int v_{i}^{(p) *} v_{i}^{(q)} \mathrm{d} V \\
& \langle p \mid q\rangle_{i j k l}=\int \varepsilon_{i j}^{(p) *} \varepsilon_{k l}^{(q)} \mathrm{d} V
\end{aligned}
$$

and the matrix elements, $\langle p \mid q\rangle_{i j k l}$, satisfy the following relationships

$$
\begin{aligned}
& \langle q \mid p\rangle_{k l i j}=\langle p \mid q\rangle_{i j k l} * \\
& \langle p \mid q\rangle_{i j k l}=\langle p \mid q\rangle_{j i k l}=\langle p \mid q\rangle_{i j l k}=\langle p \mid q\rangle_{j i l k} .
\end{aligned}
$$

It is convenient to represent the fourth-order elastic tensor $C_{i j k l}$ by the Voigt notation $C_{i j}(i, j=1,2, \cdots, 6)$ based on the conversion rules: $11 \rightarrow 1,23 \rightarrow 4$, etc. When using these conventional representations and rearranging Eq. (14) with respect to $\delta C_{i j}(1 \leq i \leq j \leq 6)$ with the aid of symmetric relation $C_{i j}=C_{j i}$, the change $\delta \omega$ in eigenfrequency is expressed by

$$
\delta \omega=\frac{\sum_{i=1}^{6} \sum_{j=i}^{6} \delta C_{i j} \sum_{p=1}^{N} \sum_{q=1}^{N}\langle p \mid q\rangle_{i j} a_{p}{ }^{*} a_{q}-\omega^{2} \delta \varrho \sum_{p=1}^{N} \sum_{q=1}^{N}\langle p \mid q\rangle a_{p}{ }^{*} a_{q}}{2 \omega \varrho \sum_{p=1}^{N} \sum_{q=1}^{N}\langle p \mid q\rangle a_{p}{ }^{*} a_{q}},
$$

where the relationships between $\langle p \mid q\rangle_{i j}$ and $\langle p \mid q\rangle_{i j k l}$ are given in Appendix A. Since the $\delta \omega$ is expressed as a linear function of $\delta C_{i j}$ and $\delta Q$, the partial derivatives of the eigenfrequency are easily written as

$$
\begin{gathered}
\partial \omega / \partial \varrho=-\omega /(2 \varrho) \\
\partial \omega / \partial C_{i j}=\Lambda_{i j} / 2 \omega \varrho \sum_{p=1}^{N} \sum_{q=1}^{N}\langle p \mid q\rangle a_{p}{ }^{*} a_{q},
\end{gathered}
$$

where

$$
\Lambda_{i j}=\sum_{p=1}^{N} \sum_{q=1}^{N}\langle p \mid q\rangle_{i j} a_{p}{ }^{*} a_{q} .
$$

The eigenfrequency $\omega$ and the eigenvector $a_{p}$ are determined by solving Eq. (6), and $\langle p \mid q\rangle$ and $\langle p \mid q\rangle_{i j}$ are also calculated by Eq. (15) with the aid of Appendix A. Then the partial derivatives can be calculated by Eqs. (18) and (19). 


\section{Analytic Expression of $\Lambda_{i j}$}

In this section, analytic expression of $\Lambda_{i j}$ in Eq. (19) is derived for rectangular parallelepiped and sphere of homogeneous and anisotropic materials.

\subsection{Rectangular parallelepiped}

In order to solve problem on free oscillation of a rectangular parallelepiped specimen with edge lengths $2 L_{i}(i=1,2,3)$, Demarest (1971) and Ohno (1976) expanded the $i$-th component $U_{i}$ of displacement vector in the Cartesian coordinates as follows:

$$
U_{i}=\sum_{p=1}^{N} a_{p}^{i} v^{(p)}
$$

with

$$
v^{(p)}=P_{l}\left(x_{1} / L_{1}\right) P_{m}\left(x_{2} / L_{2}\right) P_{n}\left(x_{3} / L_{3}\right) / \sqrt{L_{1} L_{2} L_{3}},
$$

where $P_{l}(x)$ is the normalized Legendre polynomial of the $l$-th order. Thus the superscript " $p$ " of $v^{(p)}$ is specified by a combination of indices $(l, m, n)$. Because of the orthogonality of the basis functions and the condition given by Eq. (8), the denominator in Eq. (18b) is reduced to

$$
\sum_{p=1}^{N} \sum_{q=1}^{N}\langle p \mid q\rangle a_{p}^{*} a_{q}=\sum_{i=1}^{3} \sum_{p=1}^{N}\left(a_{p}^{i}\right)^{*}\left(a_{p}^{i}\right)=1 .
$$

On the other hand, the change in strain energy due to small change of elastic constants $\delta C_{i j}$ is written as

$$
\sum_{p=1}^{N} \sum_{q=1}^{N}\langle p|\delta C| q\rangle a_{p}{ }^{*} a_{q}=\sum_{s=1}^{3} \sum_{t=1}^{3} \sum_{p=1}^{N} \sum_{q=1}^{N}\left(\Gamma_{p q}\right)^{s t}\left(a_{p}^{s}\right)^{*}\left(a_{q}^{t}\right)=\sum_{i=1}^{6} \sum_{j=i}^{6} \delta C_{i j} \Lambda_{i j},
$$

where the matrix elements $\left(\Gamma_{p q}\right)^{s t}$ are given in Appendix B. Substituting the matrix elements into Eq. (23) and rearranging with respect to $\delta C_{i j}$, we obtain $\Lambda_{i j}(1 \leq i \leq j \leq 6)$ as follows

$$
\begin{aligned}
& \Lambda_{11}=\sum_{p=1}^{N} \sum_{q=1}^{N} G_{1}\left(a_{p}^{1}\right)^{*}\left(a_{q}^{1}\right) \\
& \Lambda_{12}=\sum_{p=1}^{N} \sum_{q=1}^{N}\left\{G_{8}\left(a_{p}^{2}\right)^{*}\left(a_{q}^{1}\right)+G_{9}\left(a_{p}^{1}\right)^{*}\left(a_{q}^{2}\right)\right\} \\
& \Lambda_{13}=\sum_{p=1}^{N} \sum_{q=1}^{N}\left\{G_{6}\left(a_{p}^{1}\right)^{*}\left(a_{q}^{3}\right)+G_{7}\left(a_{p}^{3}\right)^{*}\left(a_{q}^{1}\right)\right\} \\
& \Lambda_{14}=\sum_{p=1}^{N} \sum_{q=1}^{N}\left\{G_{6}\left(a_{p}^{1}\right)^{*}\left(a_{q}^{2}\right)+G_{7}\left(a_{p}^{2}\right)^{*}\left(a_{q}^{1}\right)+G_{8}\left(a_{p}^{3}\right)^{*}\left(a_{q}^{1}\right)+G_{9}\left(a_{p}^{1}\right)^{*}\left(a_{q}^{3}\right)\right\} \\
& \Lambda_{15}=\sum_{p=1}^{N} \sum_{q=1}^{N}\left[G_{1}\left\{\left(a_{p}^{1}\right)^{*}\left(a_{q}^{3}\right)+\left(a_{p}^{3}\right)^{*}\left(a_{q}^{1}\right)\right\}+\left(G_{6}+G_{7}\right)\left(a_{p}^{1}\right)^{*}\left(a_{q}^{1}\right)\right]
\end{aligned}
$$




$$
\begin{aligned}
& \Lambda_{16}=\sum_{p=1}^{N} \sum_{q=1}^{N}\left[G_{1}\left\{\left(a_{p}^{1}\right)^{*}\left(a_{q}^{2}\right)+\left(a_{p}^{2}\right)^{*}\left(a_{q}^{1}\right)\right\}+\left(G_{8}+G_{9}\right)\left(a_{p}^{1}\right)^{*}\left(a_{q}^{1}\right)\right] \\
& \Lambda_{22}=\sum_{p=1}^{N} \sum_{q=1}^{N} G_{2}\left(a_{p}^{2}\right)^{*}\left(a_{q}^{2}\right) \\
& \Lambda_{23}=\sum_{p=1}^{N} \sum_{q=1}^{N}\left\{G_{4}\left(a_{p}^{3}\right)^{*}\left(a_{q}^{2}\right)+G_{5}\left(a_{p}^{2}\right)^{*}\left(a_{q}^{3}\right)\right\} \\
& \Lambda_{24}=\sum_{p=1}^{N} \sum_{q=1}^{N}\left[G_{2}\left\{\left(a_{p}^{2}\right)^{*}\left(a_{q}^{3}\right)+\left(a_{p}^{3}\right)^{*}\left(a_{q}^{2}\right)\right\}+\left(G_{4}+G_{5}\right)\left(a_{p}^{2}\right)^{*}\left(a_{q}^{2}\right)\right] \\
& \Lambda_{25}=\sum_{p=1}^{N} \sum_{q=1}^{N}\left\{G_{4}\left(a_{p}^{1}\right)^{*}\left(a_{q}^{2}\right)+G_{5}\left(a_{p}^{2}\right)^{*}\left(a_{q}^{1}\right)+G_{8}\left(a_{p}^{2}\right)^{*}\left(a_{q}^{3}\right)+G_{9}\left(a_{p}^{3}\right)^{*}\left(a_{q}^{2}\right)\right\} \\
& \Lambda_{26}=\sum_{p=1}^{N} \sum_{q=1}^{N}\left[G_{2}\left\{\left(a_{p}^{1}\right)^{*}\left(a_{q}^{2}\right)+\left(a_{p}^{2}\right)^{*}\left(a_{q}^{1}\right)\right\}+\left(G_{8}+G_{9}\right)\left(a_{p}^{2}\right)^{*}\left(a_{q}^{2}\right)\right] \\
& \Lambda_{33}=\sum_{p=1}^{N} \sum_{q=1}^{N} G_{3}\left(a_{p}^{3}\right)^{*}\left(a_{q}^{3}\right) \\
& \Lambda_{34}=\sum_{p=1}^{N} \sum_{q=1}^{N}\left[G_{3}\left\{\left(a_{p}^{2}\right)^{*}\left(a_{q}^{3}\right)+\left(a_{p}^{3}\right)^{*}\left(a_{q}^{2}\right)\right\}+\left(G_{4}+G_{5}\right)\left(a_{p}^{3}\right)^{*}\left(a_{q}^{3}\right)\right] \\
& \Lambda_{35}=\sum_{p=1}^{N} \sum_{q=1}^{N}\left[G_{3}\left\{\left(a_{p}^{1}\right)^{*}\left(a_{q}^{3}\right)+\left(a_{p}^{3}\right)^{*}\left(a_{q}^{1}\right)\right\}+\left(G_{6}+G_{7}\right)\left(a_{p}^{2}\right)^{*}\left(a_{q}^{2}\right)\right] \\
& \Lambda_{36}=\sum_{p=1}^{N} \sum_{q=1}^{N}\left\{G_{4}\left(a_{p}^{3}\right)^{*}\left(a_{q}^{1}\right)+G_{5}\left(a_{p}^{1}\right)^{*}\left(a_{q}^{3}\right)+G_{6}\left(a_{p}^{2}\right)^{*}\left(a_{q}^{3}\right)+G_{7}\left(a_{p}^{3}\right)^{*}\left(a_{q}^{2}\right)\right\} \\
& \Lambda_{44}=\sum_{p=1}^{N} \sum_{q=1}^{N}\left\{G_{2}\left(a_{p}^{3}\right)^{*}\left(a_{q}^{3}\right)+G_{3}\left(a_{p}^{2}\right)^{*}\left(a_{q}^{2}\right)+G_{4}\left(a_{p}^{2}\right)^{*}\left(a_{q}^{3}\right)+G_{5}\left(a_{p}^{3}\right)^{*}\left(a_{q}^{2}\right)\right\} \\
& \Lambda_{45}=\sum_{p=1}^{N} \sum_{q=1}^{N}\left[G_{3}\left\{\left(a_{p}^{1}\right)^{*}\left(a_{q}^{2}\right)+\left(a_{p}^{2}\right)^{*}\left(a_{q}^{1}\right)\right\}+G_{4}\left(a_{p}^{1}\right)^{*}\left(a_{q}^{3}\right)+G_{5}\left(a_{p}^{3}\right)^{*}\left(a_{q}^{1}\right)\right. \\
& \left.+G_{6}\left(a_{p}^{3}\right)^{*}\left(a_{q}^{2}\right)+G_{7}\left(a_{p}^{2}\right)^{*}\left(a_{q}^{3}\right)+\left(G_{8}+G_{9}\right)\left(a_{p}^{3}\right)^{*}\left(a_{q}^{3}\right)\right] \\
& A_{46}=\sum_{p=1}^{N} \sum_{q=1}^{N}\left[G_{2}\left\{\left(a_{p}^{3}\right)^{*}\left(a_{q}^{1}\right)+\left(a_{p}^{1}\right)^{*}\left(a_{q}^{3}\right)\right\}+G_{4}\left(a_{p}^{2}\right)^{*}\left(a_{q}^{1}\right)+G_{5}\left(a_{p}^{1}\right)^{*}\left(a_{q}^{2}\right)\right. \\
& \left.+\left(G_{6}+G_{7}\right)\left(a_{p}^{2}\right)^{*}\left(a_{q}^{2}\right)+G_{8}\left(a_{p}^{3}\right)^{*}\left(a_{q}^{2}\right)+G_{9}\left(a_{p}^{2}\right)^{*}\left(a_{q}^{3}\right)\right] \\
& \Lambda_{55}=\sum_{p=1}^{N} \sum_{q=1}^{N}\left\{G_{1}\left(a_{p}^{3}\right)^{*}\left(a_{q}^{3}\right)+G_{3}\left(a_{p}^{1}\right)^{*}\left(a_{q}^{1}\right)+G_{6}\left(a_{p}^{3}\right)^{*}\left(a_{q}^{1}\right)+G_{7}\left(a_{p}^{1}\right)^{*}\left(a_{q}^{3}\right)\right\} \\
& \Lambda_{56}=\sum_{p=1}^{N} \sum_{q=1}^{N}\left[G_{1}\left\{\left(a_{p}^{2}\right)^{*}\left(a_{q}^{3}\right)+\left(a_{p}^{3}\right)^{*}\left(a_{q}^{2}\right)\right\}+\left(G_{4}+G_{5}\right)\left(a_{p}^{1}\right)^{*}\left(a_{q}^{1}\right)+G_{6}\left(a_{p}^{2}\right)^{*}\left(a_{q}^{1}\right)\right. \\
& \left.+G_{7}\left(a_{p}^{1}\right)^{*}\left(a_{q}^{2}\right)+G_{8}\left(a_{p}^{1}\right)^{*}\left(a_{q}^{3}\right)+G_{9}\left(a_{p}^{3}\right)^{*}\left(a_{q}^{1}\right)\right]
\end{aligned}
$$

Vol. 41, No. 5, 1993 


$$
\Lambda_{66}=\sum_{p=1}^{N} \sum_{q=1}^{N}\left\{G_{1}\left(a_{p}^{2}\right)^{*}\left(a_{q}^{2}\right)+G_{2}\left(a_{p}^{1}\right)^{*}\left(a_{q}^{1}\right)+G_{8}\left(a_{p}^{1}\right)^{*}\left(a_{q}^{2}\right)+G_{9}\left(a_{p}^{2}\right)^{*}\left(a_{q}^{1}\right)\right\},
$$

where the coefficients $G_{i}(i=1,2, \cdots, 9)$ are given by Ohno (1976).

\subsection{Sphere}

In order to describe free oscillation of an elastic sphere, it is convenient to use the contravariant canonical components, $u^{\alpha}, \varepsilon^{\alpha \beta}$, and $K^{\alpha \beta \gamma \delta}$, of displacement vector, strain tensor and fourth-order elastic tensor in spherical coordinates (cf., Phinney and Burridge, 1973)

$$
\begin{gathered}
u^{\alpha}=C_{\alpha i}^{+} u_{i} \\
\varepsilon^{\alpha \beta}=C_{\alpha i}^{+} C_{\beta j}^{+} \varepsilon_{i j} \\
K^{\alpha \beta \gamma \delta}=C_{\alpha i}^{+} C_{\beta j}^{+} C_{\gamma k}^{+} C_{\delta_{l}}^{+} K_{i j k l},
\end{gathered}
$$

where the Greek indices take one of indices $(-1,0,1)$ (abbreviated to $-, 0,+)$, the subscripts $i, j, k$, and $l$ take one of directional indices $(1,2,3)$ corresponding to $(\theta, \phi, r)$, and $C_{\alpha i}^{+}$is the Hermitian conjugate of a unitary matrix

$$
C_{i \alpha}=\left[\begin{array}{ccc}
1 / \sqrt{2} & 0 & -1 / \sqrt{2} \\
-i / \sqrt{2} & 0 & -i / \sqrt{2} \\
0 & 1 & 0
\end{array}\right],
$$

where $i=\sqrt{-1}$. The elastic tensor $K_{i j k l}$ in spherical coordinates is related to that $C_{i^{\prime} j^{\prime} k^{\prime} l^{\prime}}$ in Cartesian coordinates by

$$
K_{i j k l}=T_{i i i^{\prime}} T_{j j^{\prime}} T_{k k^{\prime}} T_{l l^{\prime}} C_{i^{\prime} j^{\prime} k^{\prime} l^{\prime}},
$$

where $i^{\prime}, j^{\prime}, k^{\prime}$, and $l^{\prime}$ take one of directional indices $(1,2,3)$ corresponding to $(x, y, z)$ and $T_{i i}$ is an element of the following matrix

$$
T_{i i^{\prime}}=\left[\begin{array}{ccc}
\cos \theta \cos \phi & \cos \theta \sin \phi & -\sin \theta \\
-\sin \phi & \cos \phi & 0 \\
\sin \theta \cos \phi & \sin \theta \sin \phi & \cos \theta
\end{array}\right] \text {. }
$$

Using Eqs. (25c) and (27), Mochizuki (1988) expanded the elastic tensor $K^{\alpha \beta \gamma \delta}$ by the generalized spherical harmonics $Y_{s}^{M t}(\theta, \phi)$

$$
K^{\alpha \beta \gamma \delta}=C_{i j k l} \sum_{s=(0,2,4)} \sum_{t=-s}^{s} W_{i j k l}^{\alpha \beta \gamma \delta}(s, t) Y_{s}^{M t}(\theta, \phi),
$$

where $M=\alpha+\beta+\gamma+\delta$ and 


$$
\begin{aligned}
W_{i j k l}^{\alpha \beta \gamma \delta}(s, t)= & \sum_{\alpha^{\prime}=-1}^{1} \sum_{\beta^{\prime}=-1}^{1} \sum_{\gamma^{\prime}=-1}^{1} \sum_{\delta^{\prime}=-1}^{1} C_{\alpha^{\prime} i}^{+} C_{\beta^{\prime} j}^{+} C_{\gamma^{\prime} k}^{+} C_{\delta^{\prime} l}^{+}(2 s+1) \\
& \left(2 s_{1}+1\right)\left(2 s_{2}+1\right)\left(\begin{array}{ccc}
s_{1} & 1 & 1 \\
-(\alpha+\beta) & \alpha & \beta
\end{array}\right)\left(\begin{array}{ccc}
s_{1} & 1 & 1 \\
-\left(\alpha^{\prime}+\beta^{\prime}\right) & \alpha^{\prime} & \beta^{\prime}
\end{array}\right)\left(\begin{array}{ccc}
s_{2} & 1 & 1 \\
-(\gamma+\delta) & \gamma & \delta
\end{array}\right) \\
& \times\left(\begin{array}{ccc}
s_{2} & 1 & 1 \\
-\left(\gamma^{\prime}+\delta^{\prime}\right) & \gamma^{\prime} & \delta^{\prime}
\end{array}\right)\left(\begin{array}{ccc}
s & s_{2} \\
-(\alpha+\beta+\gamma+\delta) & \alpha+\beta & \gamma+\delta
\end{array}\right) \\
& \times\left(\begin{array}{cccc}
s & s_{1} & s_{2} \\
-t & \alpha^{\prime}+\beta^{\prime} & \gamma^{\prime}+\delta^{\prime}
\end{array}\right)
\end{aligned}
$$

where

$$
\left(\begin{array}{lll}
j_{1} & j_{2} & j_{3} \\
m_{1} & m_{2} & m_{3}
\end{array}\right)
$$

is Wigner's 3-j symbol (Edmonds, 1960), and its characteristics are shown in Appendix $\mathrm{C}$.

When an elastic sphere with radius $\hat{a}$ vibrates, the energy equation is expressed as

$$
\sum_{p=1}^{N} \sum_{q=1}^{N}\left(\varrho \omega^{2} \hat{a}^{2}\langle p \mid q\rangle-C_{i j k l}\langle p \mid q\rangle_{i j k l}\right) a_{p}{ }^{*} a_{q}=0 .
$$

When using the contravariant canonical components of displacement vectors, strains and elastic constants, we obtain the matrix elements $\langle p \mid q\rangle$ and $\langle p \mid q\rangle_{i j k l}$ as follows

$$
\begin{aligned}
& \langle p \mid q\rangle=\sum_{\alpha=-1}^{1} \int v^{\alpha(p) *} v^{\alpha(q)} \mathrm{d} V \\
\langle p \mid q\rangle_{i j k l}= & \sum_{\alpha=-1}^{1} \sum_{\beta=-1}^{1} \sum_{\gamma=-1}^{1} \sum_{\delta=-1}^{1} \sum_{s=(0,2,4)} \sum_{t=-s}^{s} W_{i j k l}^{\alpha \beta \gamma \delta}(s, t) \\
& \times \int \varepsilon^{\alpha \beta(p) *} Y_{s}^{M t}(\theta, \phi) \varepsilon^{-\gamma-\delta(q)}(-1)^{\gamma+\delta} \mathrm{d} V,
\end{aligned}
$$

where the volume integrations should be made on unit sphere.

As the basis functions in the Rayleigh-Ritz method, we adopted the eigenfunctions of free oscillation of a homogeneous and isotropic sphere, e.g., toroidal and spheroidal eigenfunctions, $\mid T, n, l, m>$ and $|S, n, l, m\rangle$, where $n, l$, and $m$ denote the radial, angular and azimuthal order numbers, respectively. Thus the superscript " $p$ " of $v^{(p)}$ in Eq. (5) is determined by a combination of $(O, n, l, m)$, where $O$ denotes $S$ or $T$ for spheroidal or toroidal eigenfunction, respectively. According to Woodhouse (1980), the contravariant canonical components of such eigenfunctions and strain tensor are generally expressed by

$$
\begin{gathered}
v^{\alpha(p)}={ }_{n} A_{l}^{\alpha}(r) Y_{l}^{\alpha m}(\theta, \phi) \\
\varepsilon^{\alpha \beta(p)}={ }_{n} B_{l}^{\alpha \beta}(r) Y_{l}^{(\alpha+\beta) m}(\theta, \phi),
\end{gathered}
$$

Vol. 41, No. 5, 1993 
where the summation convention is not applied to the repeated Greek indices and index "l," and

$$
\begin{aligned}
& { }_{n} A_{l}{ }^{0}(r)={ }_{n} U_{l}(r) \gamma_{l} \\
& { }_{n} A_{l}^{ \pm}(r)=\gamma_{l} \Omega_{0}{ }^{l}\left\{{ }_{n} V_{l}(r) \pm i_{n} W_{l}(r)\right\} \\
& { }_{n} B_{l}^{00}(r)={ }_{n} \dot{U}_{l}(r) \gamma_{l} \\
& { }_{n} B_{l}^{+-}(r)=-(1 / 2)_{n} F_{l}(r) \gamma_{l} \\
& \left.{ }_{n} B_{l}^{0 \pm}(r)=(1 / 2) \gamma_{l} \Omega_{0}{ }^{l}{ }_{n} X_{l}(r) \pm i_{n} Z_{l}(r)\right\} \\
& \left.{ }_{n} B_{l}^{ \pm \pm}(r)=\gamma_{l} \Omega_{0}{ }^{l} \Omega_{2}{ }^{l}{ }_{n} V_{l}(r) \pm i_{n} W_{l}(r)\right\} / r
\end{aligned}
$$

and

$$
\begin{aligned}
& { }_{n} F_{l}(r)=\left\{2_{n} U_{l}(r)-l(l+1)_{n} V_{l}(r)\right\} / r \\
& { }_{n} X_{l}(r)={ }_{n} \dot{V}_{l}(r)+\left\{{ }_{n} U_{l}(r)-{ }_{n} V_{l}(r)\right\} / r \\
& { }_{n} Z_{l}(r)={ }_{n} \dot{W}_{l}(r)-{ }_{n} W_{l}(r) / r \\
& \gamma_{l}=\sqrt{(2 l+1) / 4 \pi} \\
& \Omega_{N}{ }^{l}=\sqrt{(l+N)(l-N+1) / 2} .
\end{aligned}
$$

The overdot in Eqs. (35) and (36) denotes differentiation with respect to $r$, and the ${ }_{n} U_{l}(r){ }_{n} V_{l}(r)$, and ${ }_{n} W_{l}(r)$ are radial eigenfunctions of free oscillation eigenfunctions of isotropic sphere. In the expressions of ${ }_{n} A_{l}^{\alpha}(r)$ and ${ }_{n} B_{l}^{\alpha \beta}(r)$, the spheroidal and toroidal eigenfunctions are gathered, and the real and imaginary parts of them come from spheroidal and toroidal basis functions, respectively.

Introducing Eq. (33) into Eq. (32a), and using orthogonality of the generalized spherical harmonics (see Appendix C), we have

$$
\langle p \mid q\rangle=\left\langle O, n, l, m \mid O^{\prime}, n^{\prime}, l^{\prime}, m^{\prime}\right\rangle=I \delta_{p q},
$$

where $\delta_{p q}$ is Kronecker's delta, and

$$
I=\int\left[\left\{_{n} U_{l}(r)\right\}^{2}+l(l+1)\left\{_{n} V_{l}(r)\right\}^{2}\right] r^{2} \mathrm{~d} r
$$

for spheroidal eigenfunctions and

$$
I=\int l(l+1)\left\{_{n} W_{l}(r)\right\}^{2} r^{2} \mathrm{~d} r
$$

for toroidal eigenfunctions. In the matrix elements $\langle p \mid q\rangle_{i j k l}$ of strain energy obtained by substitution of Eq. (34) into Eq. (32b), on the other hand, a surface integral of triple products of the generalized spherical harmonics appears. Since this integral is analytically performed (see Appendix C), the matrix element of strain energy is expressed as 


$$
\begin{aligned}
& \langle p \mid q\rangle_{i j k l}=\left\langle O, n, l, m \mid O^{\prime}, n^{\prime}, l^{\prime}, m^{\prime}\right\rangle_{i j k l}=\sum_{\alpha=-1}^{1} \sum_{\beta=-1}^{1} \sum_{\gamma=-1}^{1} \sum_{\delta=-1}^{1} \sum_{s=(0,2,4)} W_{i j k l}^{\alpha \beta \gamma \delta}\left(s, m-m^{\prime}\right) \\
& \times 4 \pi(-1)^{m}(-1)^{M}\left(\begin{array}{ccc}
l & s & l^{\prime} \\
-m & m-m^{\prime} & m^{\prime}
\end{array}\right)\left(\begin{array}{ccc}
l & s & l^{\prime} \\
-(\alpha+\beta) & \alpha+\beta+\gamma+\delta & -(\gamma+\delta)
\end{array}\right) \\
& \times \int\left\{{ }_{n} B_{l}^{\alpha \beta}(r)\right\}^{*}{ }_{n^{\prime}} B_{l^{\prime}}{ }^{-\gamma-\delta}(r) r^{2} \mathrm{~d} r .
\end{aligned}
$$

The integrations with respect to radius in Eqs. (38) and (39) should be made in the range of $0 \leq r \leq 1$. Once the matrix elements $\langle p \mid q\rangle$ and $\langle p \mid q\rangle_{i j k l}$ are evaluated by Eqs. (37) and (39), the $\Lambda_{i j}$ is obtained from Eq. (19) through the conversion rule between $\langle p \mid q\rangle_{i j k l}$ and $\langle p \mid q\rangle_{i j}$ in Appendix A, and the derivatives of eigenfrequency with respect to $C_{i j}$ are computed by

$$
\partial \omega / \partial C_{i j}=\Lambda_{i j} / 2 \omega \varrho \hat{a}^{2} \sum_{p=1}^{N} \sum_{q=1}^{N}\langle p \mid q\rangle a_{p}{ }^{*} a_{q} .
$$

\section{Partial Derivatives with Respect to Size of Specimen}

The eigenfrequencies depend not only on elastic constants but also on sample size, as seen in Eq. (1). Both sample size and density change with the change of physical condition such as temperature, but mass $M$ of the sample is usually constant. Thus it is convenient to take density as dependent parameter. When the eigenfrequency is considered to be a function of elastic constants, mass, and size, the partial derivatives of eigenfrequency with respect to half edge lengths $L_{i}(i=1,2,3)$ of a rectangular parallelepiped are given by

$$
\left(\partial \omega / \partial L_{i}\right)_{M, C, L}=\left(\partial \omega / \partial L_{i}\right)_{\varrho, C, L}+(\partial \omega / \partial \varrho)_{C}\left(\partial \varrho / \partial L_{i}\right)_{M, L},
$$

where the $L$ and $C$ are symbolic expression of size (except $i$-th edge length) and elastic constants. For a sphere with radius $\hat{a}$, on the other hand, we have

$$
(\partial \omega / \partial \hat{a})_{M, C}=(\partial \omega / \partial \hat{a})_{\varrho, C}+(\partial \omega / \partial \varrho)_{C}(\partial \varrho / \partial \hat{a})_{M} .
$$

Since the $\left(\partial \varrho / \partial L_{i}\right)_{M, L}$ and $(\partial \varrho / \partial \hat{a})_{M}$ are written as

$$
\begin{gathered}
\left(\partial \varrho / \partial L_{i}\right)_{M, L}=-\varrho / L_{i} \\
(\partial \varrho / \partial \hat{a})_{M}=-3 \varrho / \hat{a}
\end{gathered}
$$

Eqs. (41a) and (41b) are reduced to

$$
\begin{aligned}
\left(\partial \omega / \partial L_{i}\right)_{M, C, L} & =\left(\partial \omega / \partial L_{i}\right)_{Q, C, L}+(1 / 2)\left(\omega / L_{i}\right) \\
(\partial \omega / \partial \hat{a})_{M, C} & =(\partial \omega / \partial \hat{a})_{\varrho, C}+(3 / 2)(\omega / \hat{a}) .
\end{aligned}
$$

Thus the derivatives, $\left(\partial \omega / \partial L_{i}\right)_{\varrho, C, L}$ and $(\partial \omega / \partial \hat{a})_{\varrho, C}$, should be evaluated.

\subsection{Rectangular parallelepiped}

Suppose that a half edge length $L_{i}(i=1,2,3)$ of the specimen changes by $\delta L_{i}$, 
keeping density $\varrho$ and elastic moduli $C_{i j}$ constant. When the higher-order terms of $\delta L_{i} / L_{i}$ are ignored, the changes of coefficients $G_{i}$ in Eq. (24) due to $\delta L_{i}$ are approximated as follows

$$
\begin{array}{ll}
\delta G_{1}=-2 G_{1} \delta L_{1} / L_{1}, \quad \delta G_{2}=-2 G_{2} \delta L_{2} / L_{2}, & \delta G_{3}=-2 G_{3} \delta L_{3} / L_{3} \\
\delta G_{4}=-G_{4}\left\{\left(\delta L_{2} / L_{2}\right)+\left(\delta L_{3} / L_{3}\right)\right\}, & \delta G_{5}=-G_{5}\left\{\left(\delta L_{2} / L_{2}\right)+\left(\delta L_{3} / L_{3}\right)\right\} \\
\delta G_{6}=-G_{6}\left\{\left(\delta L_{1} / L_{1}\right)+\left(\delta L_{3} / L_{3}\right)\right\}, & \delta G_{7}=-G_{7}\left\{\left(\delta L_{1} / L_{1}\right)+\left(\delta L_{3} / L_{3}\right)\right\} \\
\delta G_{8}=-G_{8}\left\{\left(\delta L_{1} / L_{1}\right)+\left(\delta L_{2} / L_{2}\right)\right\}, & \delta G_{9}=-G_{9}\left\{\left(\delta L_{1} / L_{1}\right)+\left(\delta L_{2} / L_{2}\right)\right\} .
\end{array}
$$

Replacing $\delta C_{i j}$ and $G_{i}$ in Eq. (23) with $C_{i j}$ and $\delta G_{i}$, respectively, and considering Eq. (22), we have a variation of energy equation

$$
2 \varrho \omega \delta \omega+\sum_{i=1}^{3}\left(\delta L_{i} / L_{i}\right) \Phi_{i}=0
$$

where

$$
\begin{aligned}
\Phi_{i}= & \sum_{p=1}^{N} \sum_{q=1}^{N}\left\{[p \mid q]_{11 i}\left(a_{p}^{1}\right)^{*} a_{q}^{1}+[p \mid q]_{12 i}\left(a_{p}^{1}\right)^{*} a_{q}^{2}+[p \mid q]_{13 i}\left(a_{p}^{1}\right)^{*} a_{q}^{3}\right. \\
& +[p \mid q]_{21 i}\left(a_{p}^{2}\right)^{*} a_{q}^{1}+[p \mid q]_{22 i}\left(a_{p}^{2}\right)^{*} a_{q}^{2}+[p \mid q]_{23 i}\left(a_{p}^{2}\right)^{*} a_{q}^{3} \\
& \left.+[p \mid q]_{31 i}\left(a_{p}^{3}\right)^{*} a_{q}^{1}+[p \mid q]_{32 i}\left(a_{p}^{3}\right)^{*} a_{q}^{2}+[p \mid q]_{33 i}\left(a_{p}^{3}\right)^{*} a_{q}^{3}\right\} .
\end{aligned}
$$

The coefficients $[p \mid q]_{i j k}$ are given in Appendix D. Therefore we simply obtain

$$
\left(\partial \omega / \partial L_{i}\right)_{\varrho, C, L}=-\Phi_{i} /\left(2 \varrho \omega L_{i}\right)
$$

Substituting this into Eq. (43a), we have

$$
\left(\partial \omega / \partial L_{i}\right)_{M, C, L}=-\Phi_{i} /\left(2 \varrho \omega L_{i}\right)+(1 / 2)\left(\omega / L_{i}\right)
$$

where the summation convention is not applied to the repeated index " $i$."

\subsection{Sphere}

When the eigenfrequency of an elastic sphere changes from $\omega$ to $\omega+\delta \omega$ owing to small change $\delta \hat{a}$ of radius in keeping density and elastic moduli constant, the variation of energy equation expressed by Eq. (31) is reduced to

$$
2 \varrho \omega \hat{a}(\omega \delta \hat{a}+\delta \omega \hat{a}) \sum_{p=1}^{N} \sum_{q=1}^{N}\langle p \mid q\rangle a_{p}^{*} a_{q}=0
$$

and we easily obtain

$$
(\partial \omega / \partial \hat{a})_{\varrho, c}=-\omega / \hat{a} .
$$

Thus Eq. (43b) for a sphere is expressed as

$$
(\partial \omega / \partial \hat{a})_{M, C}=\omega /(2 \hat{a}) .
$$

This is an analytic expression of derivative of eigenfrequency with respect to radius of a sphere. 


\section{Numerical Example}

A numerical example is given for a rectangular parallelepiped olivine, whose elastic constants, density, and edge lengths are listed in Table 1. The derivatives of eigenfrequencies with respect to elastic constants are computed by using the analytic expression, Eq. (18), and they are compared in Table 2 with those calculated by the numerical differentiation method, where the derivatives of eigenfrequency are numerically calculated by using change in eigenfrequency for small change of elastic constants. Small differences are found between the derivatives computed by the analytical and numerical methods. When dependence of eigenfrequency on the elastic constants is represented by quadratic form of elastic moduli, the values of the derivatives computed by numerical and analytical methods have to agree with each other. Thus the small differences between the derivatives may come from the third- or higher-order effect of elastic constants on the eigenfrequencies. Because the derivatives can be obtained as soon as the eigenfrequency is computed, the analytic expression is also useful for iterative inversion to determine elastic constants from observed resonant frequencies.

Elastic constants of the olivine specimen are redetermined by using the derivatives computed analytically, and the determined elastic constants are compared in Table 1 with those obtained by Ohno (1976), who used the derivatives computed by the numerical method for determination of elastic constants. Although slight differences are found between the present and previous values of the elastic constants, they are within the uncertainty of elastic constants. Thus agreement between both elastic constants is good. This suggests that the derivatives of eigenfrequencies are correctly evaluated by the analytical formula.

In elasticity measurements of elastically anisotropic spheres, the analytic expression has been successfully used for rutile, periclase, $\mathrm{CaO}$ and olivine spheres (Suzuki et al.,

Table 1. List of physical parameters of the specimen, olivine-1.*

\begin{tabular}{cc}
\hline Edge length $\left(\mathrm{mm}, l_{i}=2 L_{i}\right)$ & $\begin{array}{l}l_{1}=2.745 \\
l_{2}=2.832 \\
l_{3}=2.015\end{array}$ \\
& \multicolumn{1}{c}{$\varrho=3.316$} \\
\hline Density $\left(\mathrm{g} / \mathrm{cm}^{3}\right)$ & \\
& Elastic constants $(\mathrm{GPa})$ \\
Ohno, $(1976)$ & Present results \\
$C_{11}=324 \pm 3$ & $C_{11}=322.8 \pm 2.3$ \\
$C_{22}=196 \pm 3$ & $C_{22}=197.0 \pm 1.5$ \\
$C_{33}=232 \pm 2$ & $C_{33}=232.9 \pm 1.1$ \\
$C_{44}=63.9 \pm 0.3$ & $C_{44}=63.92 \pm 0.18$ \\
$C_{55}=77.9 \pm 0.3$ & $C_{55}=77.91 \pm 0.26$ \\
$C_{66}=78.8 \pm 0.4$ & $C_{66}=79.00 \pm 0.25$ \\
$C_{23}=68.8 \pm 1.4$ & $C_{23}=70.3 \pm 1.6$ \\
$C_{31}=71.5 \pm 1.5$ & $C_{31}=71.1 \pm 1.9$ \\
$C_{12}=71.5 \pm 1.8$ & $C_{12}=71.8 \pm 2.2$ \\
\hline
\end{tabular}

* A natural olivine with approximate composition $\left(\mathrm{Mg}_{1.8} \mathrm{Fe}_{0.2} \mathrm{SiO}_{4}\right)$.

Vol. 41, No. 5, 1993 
Table 2. Comparison of values of the derivatives, $\partial f / \partial C_{i j}(\mathrm{kHz} / \mathrm{GPa})$, computed for olivine-1 by analytical and numerical methods.

\begin{tabular}{|c|c|c|c|c|c|c|c|c|c|c|}
\hline \multicolumn{2}{|c|}{ No. Mode } & \multirow{2}{*}{$\frac{\partial f / \partial C_{11}}{0.003}$} & \multirow{2}{*}{$\frac{\partial f / \partial C_{22}}{0.007}$} & \multirow{2}{*}{$\frac{\partial f / \partial C_{33}}{0.010}$} & \multirow{2}{*}{$\frac{\partial f / \partial C_{23}}{-0.012}$} & \multirow{2}{*}{$\frac{\partial f / \partial C_{31}}{-0.008}$} & \multirow{2}{*}{$\frac{\partial f / \partial C_{12}}{0.002}$} & \multirow{2}{*}{$\frac{\partial f / \partial C_{44}}{1.274}$} & \multirow{2}{*}{$\frac{\partial f / \partial C_{55}}{0.468}$} & \multirow{3}{*}{$\begin{array}{l}\partial f / \partial C_{66} \\
2.859 \\
2.90\end{array}$} \\
\hline 1 & $A_{u}-1$ & & & & & & & & & \\
\hline & EV-1 & 0.01 & 0.02 & 0.02 & -0.03 & -0.01 & -0.01 & 1.30 & 0.50 & \\
\hline \multirow[t]{2}{*}{2} & $\mathrm{~B}_{1 u^{-1}}$ & 0.145 & 2.063 & 0.091 & -0.828 & 0.214 & -1.055 & 1.658 & 0.044 & 0.003 \\
\hline & EZ-1 & 0.12 & 2.11 & 0.08 & -0.89 & 0.17 & -1.04 & 1.65 & 0.12 & 0.11 \\
\hline \multirow[t]{2}{*}{3} & $A_{u}-2$ & 0.027 & 0.090 & 0.007 & -0.033 & 0.011 & -0.087 & 3.766 & 3.104 & 0.153 \\
\hline & $E V-2$ & 0.04 & 0.10 & 0.01 & -0.05 & 0.00 & -0.10 & 3.81 & 3.13 & 0.18 \\
\hline \multirow[t]{2}{*}{4} & $B_{3 u}-1$ & 0.056 & 2.512 & 0.274 & -1.645 & 0.218 & -0.647 & 0.003 & 0.012 & 1.147 \\
\hline & $E X-1$ & 0.07 & 2.57 & 0.30 & -1.76 & 0.20 & -0.74 & 0.00 & 0.01 & 1.07 \\
\hline \multirow[t]{2}{*}{5} & $B_{1 g^{-1}}$ & 0.025 & 0.124 & 0.020 & -0.086 & -0.022 & 0.002 & 0.001 & 0.000 & 6.536 \\
\hline & $\mathrm{OZ}-1$ & 0.03 & 0.13 & 0.02 & -0.10 & -0.04 & 0.00 & 0.02 & 0.04 & 6.58 \\
\hline \multirow[t]{2}{*}{6} & $B_{3 \mathrm{~g}^{-1}}$ & 0.027 & 0.268 & 0.018 & -0.047 & -0.003 & -0.148 & 8.249 & 0.004 & 0.007 \\
\hline & OX-1 & 0.01 & 0.29 & 0.02 & -0.04 & -0.01 & -0.14 & 8.33 & -0.01 & 0.00 \\
\hline \multirow[t]{2}{*}{7} & $A_{g}-1$ & 0.259 & 3.481 & 0.348 & -2.172 & 0.569 & -1.868 & 0.000 & 0.000 & 0.000 \\
\hline & OD-1 & 0.24 & 3.57 & 0.39 & -2.34 & 0.50 & -1.86 & 0.00 & 0.00 & 0.00 \\
\hline \multirow[t]{2}{*}{8} & $\mathrm{~B}_{2 \mathrm{~g}}-1$ & 0.092 & 1.693 & 0.113 & -0.788 & 0.160 & -0.749 & 0.764 & 2.634 & 0.857 \\
\hline & $O Y-1$ & 0.10 & 1.77 & 0.13 & -0.88 & 0.14 & -0.81 & 0.70 & 2.72 & 0.89 \\
\hline \multirow[t]{2}{*}{9} & $B_{1 u}-2$ & 1.159 & 0.070 & 0.123 & -0.057 & -0.606 & -0.058 & 0.313 & 2.919 & 0.027 \\
\hline & EZ-2 & 1.21 & 0.06 & 0.12 & -0.08 & -0.69 & -0.13 & 0.33 & 2.94 & 0.13 \\
\hline \multirow[t]{2}{*}{10} & $B_{2 u^{-1}}$ & 1.284 & 0.158 & 0.248 & 0.122 & -1.090 & -0.431 & 0.056 & 0.004 & 3.147 \\
\hline & $\mathrm{EY}-1$ & 1.34 & 0.21 & 0.32 & -0.04 & -1.23 & -0.55 & 0.06 & 0.05 & 3.08 \\
\hline \multirow[t]{2}{*}{11} & $\mathrm{~B}_{2 \mathrm{~g}}-2$ & 0.064 & 0.087 & 0.022 & -0.007 & -0.042 & 0.066 & 0.181 & 7.644 & 0.095 \\
\hline & $O Y-2$ & 0.07 & 0.11 & 0.03 & -0.03 & -0.05 & 0.05 & 0.20 & 7.73 & 0.13 \\
\hline \multirow[t]{2}{*}{12} & $A_{g}-2$ & 1.229 & 1.905 & 0.652 & -1.251 & -0.901 & -1.271 & 0.034 & 0.002 & 0.281 \\
\hline & OD-2 & 1.24 & 1.88 & 0.68 & -1.26 & -0.97 & -1.37 & 0.03 & 0.00 & 0.28 \\
\hline
\end{tabular}

In the upper row, the derivative values by the present study and mode name by Mochizuki's nomenclature (1988) are indicated. In the lower row, the derivative values by the numerical differentiation method by Ohno (1976) and mode name by Ohno's nomenclature are indicated. Values of the parameters needed for calculating the derivatives are listed in Table 1.

1992a; Oda et al., 1989; Oda et al., 1992; Suzuki et al., 1992b). Thus, report on results of actual computation for spherical specimens is omitted here.

\section{Extension to the Determination of Anisotropic Dissipation Constants}

The resonant oscillation of actual Earth's materials is dissipative in energy due to 
anelasticity. Because of energy dissipation the oscillation shows not only shift in resonant frequency but also broadening of peak width on spectrum. This dissipation phenomenon has been observed on normal modes of the solid Earth and provides us with an important information in constructing its rheological model. Anelasticity of a single-crystal is not as large as that of the Earth and the shift in eigenfrequencies due to dissipation is negligibly small for attenuating oscillation of the single-crystal, but the accurate measurement of attenuation is useful to clarify the mechanisms of physical dissipation. The anelastic properties of dissipative materials are represented by complex elastic constants, $C_{i j}\left(1+i Q_{i j}{ }^{-1}\right)$, where the $Q_{i j}{ }^{-1}$ are intrinsic dissipation constants of the material, and the summation convention is not applied for repeated indices. Responding to the introduction of complex elastic constants, the eigenfrequency is expressed as complex quantity, $\omega(1+i q / 2)$, where the real part is eigenfrequency calculated from real parts of the complex elastic constants and the $q$ denotes dimensionless parameter representing degree of attenuation of oscillation. Because the imaginary part of the complex elastic constants is so small that the small change of $\delta \omega$ and $\delta C_{i j}$ in Eq. (17) are replaced by $\delta \omega=i \omega q / 2$ and $\delta C_{i j}=i C_{i j} Q_{i j}{ }^{-1}$, the vibrational attenuation, $q$, is expressed by

$$
q=\frac{\sum_{i=1}^{6} \sum_{j=i}^{6} \Lambda_{i j} C_{i j} Q_{i j}{ }^{-1}}{\varrho \omega^{2} \sum_{p=1}^{N} \sum_{q=1}^{N}\langle p \mid q\rangle a_{p}{ }^{*} a_{q}} .
$$

This means that the $q$ is expressed by a linear combination of $Q_{i j}{ }^{-1}$.

It was demonstrated that the resonance method is applicable to determination of anisotropic dissipation constants $Q_{i j}{ }^{-1}$ from vibrational attenuation data of many oscillation modes through an inversion calculation (Sumino et al., 1976). In the right-hand side of Eq. (52), the parameters other than $Q_{i j}{ }^{-1}$ are already evaluated in determining the real parts of the complex elastic constants from observed resonant frequencies. When the vibrational attenuation, $q$, is measured for a number of oscillation modes, the unknown parameters $Q_{i j}{ }^{-1}$ may be determined from the observed attenuation data by the inversion technique similar to that used to determine the elastic constants. This method has successfully applied to determination of anisotropic dissipation constants of $\mathrm{CaO}$ and olivine single-crystals (Oda et al., 1991; Suzuki et al., 1992 b).

\section{Conclusions}

The analytic expression for computation of the partial derivatives of eigenfrequency with respect to elastic constants was derived for the isotropic Earth by Takeuchi and Saito (1972), and the algorithm has been used to determine Earth's structure from seismic data on surface wave dispersions and on eigenperiods of Earth's free oscillation (e.g., Jordan and Anderson, 1974). The present work has extended their study to the free oscillation of a finite anisotropic body for the purpose of accurate determination of elastic constants by resonance method.

The present analytical method gives the following advantages: 1) the partial 
derivatives of eigenfrequencies are accurately and quickly computed, in comparison with the numerical differentiation method, and 2) iterative inversion to determine elastic constants from data of eigenfrequencies is possible, because the derivatives are calculated as soon as the eigenfrequencies are obtained.

The authors are grateful to Prof. Kumazawa for his comments on the present work and Dr. Mochizuki for reading the manuscript. They also thank Prof. Seya.for his encouragement during the present study.

\section{REFERENCES}

Babuska, V. J. F., M. Kumazawa, I. Ohno, and Y. Sumino, Elastic property of garnet solid-solution series, Phys. Earth Planet. Inter., 16, 157-176, 1978.

Demarest, H. H., Cube-resonance method to determine the elastic constants of solids, J. Acoust. Soc. Am., 49, 768-775, 1971.

Edmonds, A. R., Angular Momentum in Quantum Mechanics, Princeton Univ. Press, New Jersey, 1960.

Goto, T. and O. L. Anderson, Apparatus for measuring elastic constants of single crystals by a resonance technique up to $1825 \mathrm{~K}$, Rev. Sci. Instrum., 59, 1405-1508, 1988.

Goto, T., I. Ohno, and Y. Sumino, The determination of the elastic constants of natural almandine-pyrope garnet by rectangular parallelepiped resonance method, J. Phys. Earth, 24, 149-156, 1976.

Jordan, T. H. and D. L. Anderson, Earth structure from free oscillations and travel times, Geophys. J. R. Astron. Soc., 36, 411-45்9, 1974.

McSkimin, H. J., Ultrasonic method for measuring the mechanical properties of a liquid and solids, in Physical Acoustics, Vol. 1A, ed. by Mason, W. P., pp. 272-335, Academic Press, New York, 1964.

Mochizuki, E., The free oscillations of an anisotropic and heterogeneous earth, Geophys. J. $R$. Astron. Soc., 86, 167-176, 1986.

Mochizuki, E., The sphere resonance method to determine the elastic constants of crystal, $J$. Appl. Phys., 63, 5668-5673, 1988.

Oda, H., O. L. Anderson, and I. Suzuki, Measurements of elastic and anelastic properties of single crystal $\mathrm{CaO}$ by the resonant sphere technique, The 28th Ann. Tech. Mtg., Soc. Eng. Sci., Gainesville, Florida, esp28, 91014, 1-10, 1991.

Oda, H., O. L. Anderson, D. G. Isaak, and I. Suzuki, Measurements of elastic properties of single crystal $\mathrm{CaO}$ up to 1200K, Phys. Chem. Miner., 19, 96-105, 1992.

Oda, H., I. Suzuki, S. Isoda, and K. Seya, An application of the resonant sphere technique to determine elastic constants of single-crystal periclase, EOS Trans. Am. Geophys. Union, 70, 1368, 1989.

Ohno, I., Free vibration of a rectangular parallelepiped crystal and its application to determination of elastic constants of orthorhombic crystals, J. Phys. Earth, 24, 355-379, 1976.

Ohno, I., S. Yamamoto, O. L. Anderson, and J. Noda, Determination of elastic constants of trigonal crystals by the rectangular parallelepiped resonance method, J. Phys. Chem. Solids, 47, 1103-1108, 1986.

Phinney, R. A. and R. Burridge, Representation of the elastic-gravitational excitation of a 
spherical earth model by generalized spherical harmonics, Geophys. J. R. Astron. Soc., 34, $451-487,1973$.

Sawamoto, H., D. J. Weidner, S. Sasaki, and M. Kumazawa, Single-crystal elastic properties of the modified spinel (beta) phase of magnesium orthosilicates, Science, 24, 749-751, 1984.

Soga, N. and O. L. Anderson, Elastic properties of tektites measured by resonant sphere technique, J. Geophys. Res., 72, 1733-1739, 1967.

Sumino, Y. and O. Nishizawa, Temperature variation of elastic constants of pyrope-almandine garnets, J. Phys. Earth, 26, 239-252, 1978.

Sumino, Y., O. L. Anderson, and I. Suzuki, Temperature coefficients of elastic constants of single crystal MgO between 80 and 1,300K, Phys. Chem. Miner., 9, 38-47, 1983.

Sumino, Y., I. Ohno, T. Goto, and M. Kumazawa, Measurement of elastic constants and internal frictions on single-crystal $\mathrm{MgO}$ by rectangular parallelepiped resonance, J. Phys. Earth, 24, 263-273, 1976.

Suzuki, I., O. L. Anderson, and Y. Sumino, Elastic properties of a single-crystal forsterite $\mathrm{Mg}_{2} \mathrm{SiO}_{4}$, up to $1200 \mathrm{k}$, Phys. Chem. Miner., 10, 38-46, 1983.

Suzuki, I., H. Oda, S. Isoda, T. Saito, and K. Seya, Free oscillation of an elastically anisotropic sphere and its application to determining the elastic constants of rutile, J. Phys. Earth, 46, 601-616, 1992a.

Suzuki, I., Y. Inouye, J. Hirao, H. Oda, T. Saito, and K. Seya, The resonant sphere technique for measurements of elasticity and anelasticity of a small mineral specimen: an application to olivine, J. Seismol. Soc. Jpn., 45, 213-228, 1992b (in Japanese with English abstract).

Takeuchi, H. and M. Saito, Seismic surface waves, in Methods in Computational Physics, ed. by Bolt, B. A., pp. 217-295, Academic Press, New York, 1972.

Yamamoto, S., I. Ohno, and O. L. Anderson, High temperature elasticity of sodium chloride, J. Phys. Chem. Solids, 48, 143-151, 1987.

Weidner, D. J. and H. R. Carleton, Elasticity of microcrystals, J. Geophys. Res., 82, 1334-1346, 1977.

Woodhouse, J. H., The coupling and attenuation of nearly resonant multiplets in the earth's free oscillation spectrum, Geophys. J. R. Astron. Soc., 61, 261-283, 1980.

\section{APPENDIX A}

The relationships between $\langle p \mid q\rangle_{i j k l}$ and $\langle p \mid q\rangle_{i j}$ are given as follows:

$$
\begin{array}{ll}
\langle p \mid q\rangle_{11}=\langle p \mid q\rangle_{1111} & \langle p \mid q\rangle_{12}=\langle p \mid q\rangle_{1122}+\langle p \mid q\rangle_{2211} \\
\langle p \mid q\rangle_{13}=\langle p \mid q\rangle_{1133}+\langle p \mid q\rangle_{3311} & \langle p \mid q\rangle_{14}=2\left(\langle p \mid q\rangle_{1132}+\langle p \mid q\rangle_{3211}\right) \\
\langle p \mid q\rangle_{15}=2\left(\langle p \mid q\rangle_{1113}+\langle p \mid q\rangle_{1311}\right) & \langle p \mid q\rangle_{16}=2\left(\langle p \mid q\rangle_{1112}+\langle p \mid q\rangle_{1211}\right) \\
\langle p \mid q\rangle_{22}=\langle p \mid q\rangle_{2222} & \langle p \mid q\rangle_{23}=\langle p \mid q\rangle_{2233}+\langle p \mid q\rangle_{3322} \\
\langle p \mid q\rangle_{24}=2\left(\langle p \mid q\rangle_{2232}+\langle p \mid q\rangle_{3222}\right) & \langle p \mid q\rangle_{25}=2\left(\langle p \mid q\rangle_{2213}+\langle p \mid q\rangle_{1322}\right) \\
\langle p \mid q\rangle_{26}=2\left(\langle p \mid q\rangle_{2212}+\langle p \mid q\rangle_{1222}\right) & \langle p \mid q\rangle_{33}=\langle p \mid q\rangle_{3333} \\
\langle p \mid q\rangle_{34}=2\left(\langle p \mid q\rangle_{3332}+\langle p \mid q\rangle_{3233}\right) & \langle p \mid q\rangle_{35}=2\left(\langle p \mid q\rangle_{3313}+\langle p \mid q\rangle_{1333}\right) \\
\langle p \mid q\rangle_{36}=2\left(\langle p \mid q\rangle_{3312}+\langle p \mid q\rangle_{1233}\right) & \langle p \mid q\rangle_{44}=4\langle p \mid q\rangle_{2323} \\
\langle p \mid q\rangle_{45}=4\left(\langle p \mid q\rangle_{2313}+\langle p \mid q\rangle_{1323}\right) & \langle p \mid q\rangle_{46}=4\left(\langle p \mid q\rangle_{2312}+\langle p \mid q\rangle_{1223}\right) \\
\langle p \mid q\rangle_{55}=4\langle p \mid q\rangle_{1313} & \langle p \mid q\rangle_{56}=4\left(\langle p \mid q\rangle_{1312}+\langle p \mid q\rangle_{1213}\right) \\
\langle p \mid q\rangle_{66}=4\langle p \mid q\rangle_{1212} &
\end{array}
$$

Vol. 41, No. 5, 1993 


\section{APPENDIX B}

$\left(\Gamma_{p q}\right)^{\mathrm{1} 1}=\delta C_{11} G_{1}+\delta C_{66} G_{2}+\delta C_{55} G_{3}+\delta C_{56} G_{4}+\delta C_{56} G_{5}+\delta C_{15} G_{6}+\delta C_{15} G_{7}+\delta C_{16} G_{8}+\delta C_{16} G_{9}$ $\left(\Gamma_{p q}\right)^{22}=\delta C_{66} G_{1}+\delta C_{22} G_{2}+\delta C_{44} G_{3}+\delta C_{24} G_{4}+\delta C_{24} G_{5}+\delta C_{46} G_{6}+\delta C_{46} G_{7}+\delta C_{26} G_{8}+\delta C_{26} G_{9}$ $\left(\Gamma_{p q}\right)^{33}=\delta C_{55} G_{1}+\delta C_{44} G_{2}+\delta C_{33} G_{3}+\delta C_{34} G_{4}+\delta C_{34} G_{5}+\delta C_{35} G_{6}+\delta C_{35} G_{7}+\delta C_{45} G_{8}+\delta C_{45} G_{9}$ $\left(\Gamma_{p q}\right)^{23}=\delta C_{56} G_{1}+\delta C_{24} G_{2}+\delta C_{34} G_{3}+\delta C_{44} G_{4}+\delta C_{23} G_{5}+\delta C_{36} G_{6}+\delta C_{45} G_{7}+\delta C_{25} G_{8}+\delta C_{46} G_{9}$ $\left(\Gamma_{p q}\right)^{32}=\delta C_{56} G_{1}+\delta C_{24} G_{2}+\delta C_{34} G_{3}+\delta C_{23} G_{4}+\delta C_{44} G_{5}+\delta C_{45} G_{6}+\delta C_{36} G_{7}+\delta C_{46} G_{8}+\delta C_{25} G_{9}$ $\left(\Gamma_{p q}\right)^{31}=\delta C_{15} G_{1}+\delta C_{46} G_{2}+\delta C_{35} G_{3}+\delta C_{36} G_{4}+\delta C_{45} G_{5}+\delta C_{55} G_{6}+\delta C_{13} G_{7}+\delta C_{14} G_{8}+\delta C_{56} G_{9}$ $\left(\Gamma_{p q}\right)^{13}=\delta C_{15} G_{1}+\delta C_{46} G_{2}+\delta C_{35} G_{3}+\delta C_{45} G_{4}+\delta C_{36} G_{5}+\delta C_{13} G_{6}+\delta C_{55} G_{7}+\delta C_{56} G_{8}+\delta C_{14} G_{9}$ $\left(\Gamma_{p q}\right)^{12}=\delta C_{16} G_{1}+\delta C_{26} G_{2}+\delta C_{45} G_{3}+\delta C_{25} G_{4}+\delta C_{46} G_{5}+\delta C_{14} G_{6}+\delta C_{56} G_{7}+\delta C_{66} G_{8}+\delta C_{12} G_{9}$ $\left(\Gamma_{p q}\right)^{21}=\delta C_{16} G_{1}+\delta C_{26} G_{2}+\delta C_{45} G_{3}+\delta C_{46} G_{4}+\delta C_{25} G_{5}+\delta C_{56} G_{6}+\delta C_{14} G_{7}+\delta C_{12} G_{8}+\delta C_{66} G_{9}$

\section{APPENDIX C}

Orthogonality of the generalized spherical harmonics is expressed by

$$
\int\left(Y_{s}^{M t}\right)^{*} Y_{s^{\prime}}{ }^{M t^{\prime}} \mathrm{d} \Omega=\frac{4 \pi \delta_{s s^{\prime}} \delta_{t t^{\prime}}}{(2 s+1)},
$$

where the integral is made on the surface of unit sphere. The integration of triple product of generalized spherical harmonics is given by

$$
\begin{aligned}
\int\left(Y_{s}{ }^{M} t\right) * Y_{s^{\prime}}{ }^{\left(M-M^{\prime \prime}\right) t^{\prime}} Y_{s^{\prime \prime}}{ }^{M^{\prime \prime} t^{\prime \prime}} \mathrm{d} \Omega=4 \pi(-1)^{M+t} & \\
& \times\left(\begin{array}{cccc}
s & s^{\prime} & s^{\prime \prime} \\
-M & M-M^{\prime \prime} & M^{\prime \prime}
\end{array}\right)\left(\begin{array}{cccc}
s & s^{\prime} & s^{\prime \prime} \\
-t & t^{\prime} & t^{\prime \prime}
\end{array}\right),
\end{aligned}
$$

where Wigner's $3-j$ symbol

$$
\left(\begin{array}{ccc}
l_{1} & l_{2} & l_{3} \\
-m_{1} & m_{2} & m_{3}
\end{array}\right)
$$

is zero unless the following selection rules are satisfied

$$
\begin{gathered}
\left|m_{i}\right| \leq l_{i} \\
\left|l_{1}-l_{2}\right| \leq l_{3} \leq l_{1}+l_{2} \\
m_{1}=m_{2}+m_{3}
\end{gathered}
$$


APPENDIX D

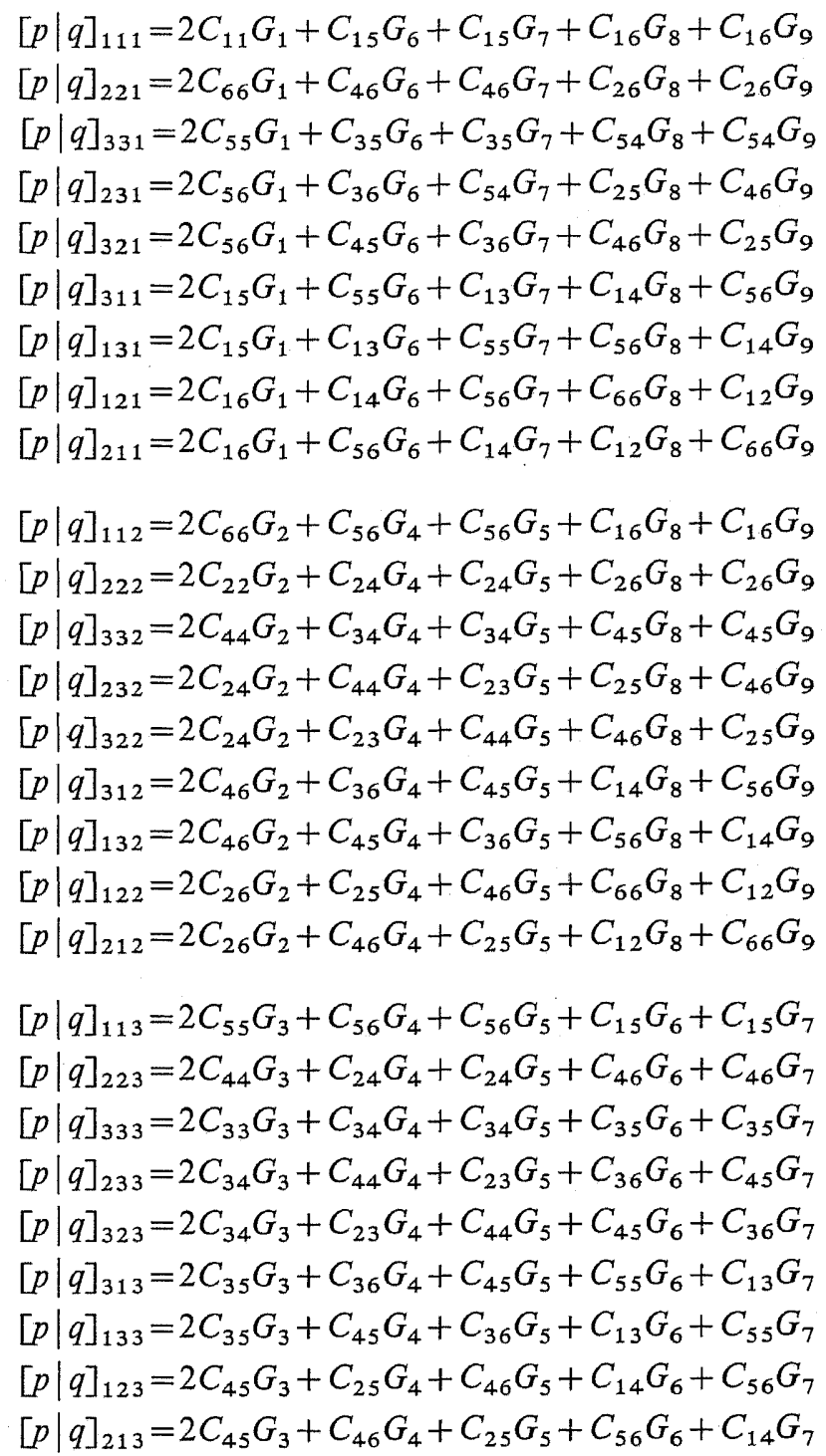

Vol. 41, No. 5, 1993 